\title{
Um Modelo Matemático Discreto para a Dispersão da Leptospirose em uma População de Ratos
}

\author{
L.A. RODRIGUES 1 , D.C. MISTRO ${ }^{2}$, Departamento de Matemática, UFSM, \\ 97105-900 Santa Maria, RS, Brasil \\ S.M.H. CENTENARO 3 , Depto. de Física, Matemática e Estatística, UNIJUí, \\ 98700-000 Ijuí, RS, Brasil.
}

\begin{abstract}
Resumo. Neste trabalho, propomos um modelo matemático para descrever a dinâmica de uma epidemia de leptospirose em uma população de ratos. O objetivo é estudar o comportamento das soluções com relação a questões essenciais no estudo de uma epidemia, tais como velocidade de propagação e o seu padrão de espalhamento espacial. Consideramos um modelo SI discreto espacialmente estruturado como um reticulado bidimensional de coordenadas inteiras. Em cada vértice desse reticulado, a população será classificada em dois estados: suscetíveis e infectados. O modelo prevê soluções estáveis, periódicas e caóticas.
\end{abstract}

Palavras-chave. Redes de Mapas Acoplados, Epidemiologia, Leptospirose.

\section{Introdução}

Nos modelos matemáticos epidemiológicos, a população é dividida em compartimentos que refletem o estado em que os indivíduos se encontram no desenvolvimento da doença, como por exemplo, suscetíveis (S), infectados (I) e recuperados (R). As características da doença determinam o tipo de modelo a ser escolhido. Nos modelos SI, não há recuperação dos indivíduos. Os suscetíveis passam à classe dos infectados pelo contato com indivíduos já infectados. Nos modelos SIS, os indivíduos infectados recuperam-se mas não adquirem imunidade e, portanto, voltam à classe dos suscetíveis. Já nos modelos SIR, os infectados recuperam-se e adquirem imunidade à doença ou morrem passando assim, para a categoria dos recuperados.

Comportamentos periódicos e caóticos são observados com freqüência em fenômenos de propagação de certas doenças. Por exemplo, alguns dados temporais observados para caxumba e catapora parecem refletir periodicidade, enquanto dados de sarampo indicam a existência de caos [8]. Tais situações não podem ser tratadas por modelos contínuos simples. Modelos contínuos descritos por equações diferenciais ordinárias com coeficientes constantes não apresentam comportamento periódico. Por outro lado, equações a diferenças, que exibem comportamentos periódicos e

\footnotetext{
${ }^{1}$ luizdiaz@smail.ufsm.br

2 dcmistro@gmail.com

${ }^{3}$ sandra-marisa@brigadamilitar.rs.gov.br
} 
caóticos para determinados valores dos parâmetros, também podem ser usadas para formular modelos epidêmicos do tipo SI, SIS ou SIR [1] exibindo comportamento periódico e caos para determinados valores dos parâmetros $[1,4]$.

A leptospirose é uma enfermidade considerada endêmica no Brasil e apresenta manifestações clínicas graves. É causada por uma bactéria do gênero Leptospira, que pode ser transmitida de animal para animal e também para o homem. Os roedores desempenham o papel de principal reservatório da doença, liberando as bactérias vivas em grande quantidade no meio ambiente, contaminando água, solos e alimentos. A infecção humana pela leptospirose resulta da exposição direta ou indireta à urina dos animais infectados presente em regiões de córregos, enchentes e plantações irrigadas, daí a importância do estudo da dinâmica da doença em uma população de ratos $[3,9]$.

A urina do portador contamina o solo úmido, seu ninho ou esconderijo e as áreas que o animal freqüenta, de modo que outros animais que coexistem no mesmo local também podem se infectar. A concentração de bactérias viáveis é geralmente elevada em urinas recém eliminadas, contudo, a viabilidade cai sensivelmente após as primeiras cinco horas, verificando-se a completa inviabilidade após 24 horas. Não há recuperação dos indivíduos infectados. Uma vez que adquirem a doença, os roedores carregam as bactérias da leptospira nos rins pelo resto da vida, liberando-as continuamente no meio ambiente. Além disso, o fato da doença não causar danos ao indivíduo portador indica que a mortalidade devido à doença pode ser desprezada. Estas considerações sugerem que um modelo SI é adequado para descrever a dinâmica desta enfermidade em uma população de ratos [3].

O objetivo deste trabalho é analisar o comportamento de um modelo SI discreto espacialmente distribuído para ser aplicado à dispersão da leptospirose em uma população de ratos $[2,6]$. A variável espacial é incluída considerando um domínio bidimensional dividido em manchas discretas denominadas sítios. Este tipo de formulação, que considera um sistema de equações a diferenças acopladas pelas dispersão, é conhecida como Rede de Mapas Acoplados ("Coupled Map Lattice") $[5,8]$.

\section{Modelo Espacialmente Homogêneo}

Inicialmente, analisaremos o comportamento do modelo espacialmente homogêneo. $\mathrm{Na}$ formulação do modelo, fazemos as seguintes hipóteses:

- a população total é constante, isto é, consideramos que a população total de ratos encontra-se em equilíbrio, de modo que a taxa de natalidade é igual à taxa de mortalidade;

- todos os indivíduos nascem suscetíveis;

- não há recuperação para os indivíduos infectados e, além disso, não há mortalidade devido à doença;

- o contágio se dá pelo contato dos indivíduos suscetíveis e infectados a uma taxa proporcional à fração de infectados na população. 
Desse modo, um modelo SI tempo-discreto, onde $S$ representa a população de indivíduos suscetíveis e $I$ a população de infectados, para uma epidemia de leptospirose em ratos, tem a seguinte forma:

$$
\begin{aligned}
S_{t+1} & =S_{t}-\alpha \frac{I_{t}}{N} S_{t}+\beta\left(N-S_{t}\right), \\
I_{t+1} & =I_{t}-\beta I_{t}+\alpha \frac{I_{t}}{N} S_{t},
\end{aligned}
$$

onde

$$
S_{t}+I_{t}=N, t=0,1,2, \ldots,
$$

$\alpha>0$ é a taxa de contato, isto é, o número médio de contatos efetivos de um indivíduo infectado em uma unidade de tempo e $\beta>0$ é a taxa de mortalidade natural, considerada igual à taxa de natalidade para manter a população total $N$ constante. O termo $\alpha \frac{I_{t}}{N} S_{t}$ representa a transferência de indivíduos da classe de suscetíveis para a dos infectados.

É importante observar que este modelo pressupõe que a população está homogeneamente distribuída, isto é, cada indivíduo tem a mesma probabilidade de ser infectado.

Para assegurar que as soluções de (2.1) e (2.2) sejam positivas e satisfaçam $S_{t}+I_{t}=N$ para quaisquer condições iniciais $S_{0}>0$ e $I_{0}>0$, algumas restrições devem ser feitas sobre os parâmetros. Segue da equação (2.2) que

$$
I_{1}=-\frac{\alpha}{N} I_{0}^{2}+I_{0}(1-\beta+\alpha)=p\left(I_{0}\right) .
$$

Assim, a parábola $p\left(I_{0}\right)$ deve satisfazer $0<p\left(I_{0}\right)<N$ para $0<I_{0}<N$. Observe que $p(0)=0$ e $p(N)=N(1-\beta)$.

A parábola assume seu valor máximo $p^{*}=\frac{N}{4 \alpha}(1-\beta+\alpha)^{2}$ em $I_{0}^{*}=\frac{N}{2 \alpha}(1-\beta+\alpha)^{2}$. Assim, $0<p\left(I_{0}\right)<N$ para $0<I_{0}<N$ se, e somente se,

$$
\begin{aligned}
& \text { (i) } \beta \leq 1 \mathrm{e} \\
& \text { (ii) } I_{0}^{*} \geq N \text { ou } \\
& \text { (iii) } I_{0}^{*}<N \text { e } p^{*}<N \text {. }
\end{aligned}
$$

Portanto, as soluções do sistema (2.1) e (2.2) são positivas e satisfazem $S_{t}+I_{t}=N$ se, e somente se,

$$
0<\beta \leq 1, \alpha<1
$$

$\mathrm{Ou}$

$$
(1-\sqrt{\alpha})^{2}<\beta \leq 1, \alpha>1
$$

Na Figura 1, toda a região sombreada no espaço dos parâmetros $(\alpha, \beta)$, corresponde às combinações dos parâmetros para os quais as soluções do sistema são positivas para qualquer condição inicial. 


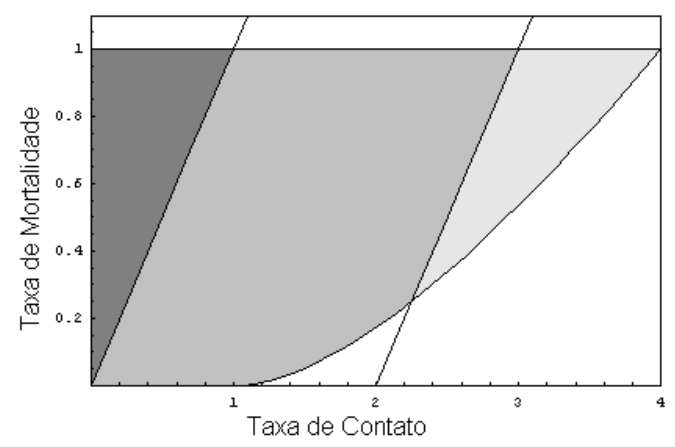

Figura 1: Região de estabilidade para os pontos de equilíbrio.

O interesse agora é explorar a estabilidade das soluções de equilíbrio do sistema (2.1) e (2.2) denotadas por $\left(S^{*}, I^{*}\right)$ :

Equilíbrio livre da doença:

$$
\left(S_{1}^{*}, I_{1}^{*}\right)=(N, 0)
$$

Equilíbrio endêmico:

$$
\left(S_{2}^{*}, I_{2}^{*}\right)=\left(\frac{\beta N}{\alpha}, N-\frac{\beta N}{\alpha}\right)
$$

É importante observar que o equilíbrio endêmico é biologicamente viável para $\alpha>\beta$.

A condição de estabilidade para $\left(S_{1}^{*}, I_{1}^{*}\right)$ é dada por

$$
\alpha<\beta<2 \text {. }
$$

No entanto, como estamos interessados somente em soluções positivas esta condição fica restrita a

$$
\alpha<\beta<1 .
$$

A região mais escura na Figura 1 representa a região de estabilidade, no espaço dos parâmetros, determinada pela condição (2.5), para o ponto de equilíbrio livre da doença.

Por outro lado, as condições de estabilidade para o ponto $\left(S_{2}^{*}, I_{2}^{*}\right)$ são:

$$
\alpha-2<\beta<\alpha \quad \text { e } \quad \beta<2 .
$$

A região de estabilidade biologicamente viável para o ponto $\left(S_{2}^{*}, I_{2}^{*}\right)$ resulta da intersecção das condições (2.3) e (2.4) com as condições de estabilidade acima:

$$
\begin{gathered}
(1-\sqrt{\alpha})^{2}<\beta<\alpha, \text { para } \alpha \leq \frac{9}{4}, \\
\alpha-2<\beta<\alpha, \text { para } \alpha>\frac{9}{4} \text { e }
\end{gathered}
$$




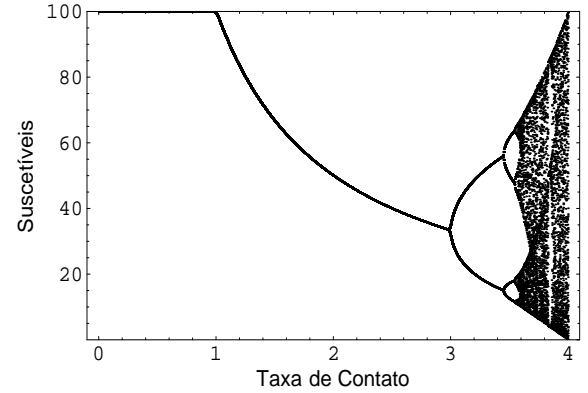

(a)

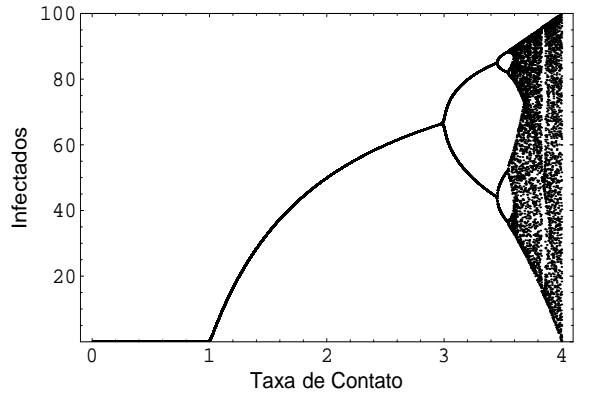

(b)

Figura 2: Diagramas de Bifurcação com relação à taxa de contato $\alpha$ para (a) Suscetíveis e (b) Infectados, para $\beta=1$.

$$
\beta \leq 1 .
$$

A região em cinza médio na Figura 1 representa a região dos parâmetros para os quais se observa estabilidade para o ponto de equilíbrio endêmico. Na região cinza claro, o estado de equilíbrio endêmico é biologicamente viável, porém instável. É a região onde ocorrem soluções periódicas e caóticas.

Na Figura 2 apresentamos diagramas de bifurcação para (a) suscetíveis e (b) infectados com relação à taxa de contato $(\alpha)$, para $\beta=1$. Podemos observar que, para $\alpha<1$, o equilíbrio livre da doença é estável. Para $\alpha>1$ o estado de equilíbrio $\left(S_{1}^{*}, I_{1}^{*}\right)$ perde a estabilidade e o equilíbrio endêmico é estável. Para valores suficientemente grandes da taxa de contato, surgem ciclos de período 2,4 , 8 e maiores até o aparecimento de soluções aperiódicas.

\section{Modelo Espacialmente Distribuído}

A dinâmica do modelo discreto espacialmente distribuído é composta de dois estágios distintos: uma fase de interação e outra de dispersão ou movimentação. Ambas as fases ocorrem em um intervalo de tempo de uma geração, sendo aplicada esta dinâmica simultaneamente a todas as células do reticulado.

O espaço é considerado como um domínio bidimensional dividido em células discretas denominadas sítios. Os sítios são regiões físicas onde os indivíduos se reproduzem sendo que a movimentação considerada é entre os sítios.

Além das hipóteses relacionadas à dinâmica da doença, consideramos que os roedores se movimentam por difusão simples. Assim, no processo de movimentação, uma fração $\mu_{I}$ da população de indivíduos infectados e uma fração $\mu_{S}$ da população de indivíduos suscetíveis, abandonam seu sítio para colonizar igualmente os quatro vizinhos mais próximos (vizinhança de Moore). As equações que caracterizam a fase de dispersão são descritas por:

$$
S_{i, j}^{\prime}=\left(1-\mu_{S}\right) S_{i, j}^{t}+\frac{\mu_{S}}{4} \sum_{v, w \in V_{i, j}} S_{v, w}^{t},
$$




$$
I_{i, j}^{\prime}=\left(1-\mu_{I}\right) I_{i, j}^{t}+\frac{\mu_{I}}{4} \sum_{v, w \in V_{i, j}} I_{v, w}^{t}
$$

onde $V_{i, j}=\{(i-1, j),(i+1, j),(i, j-1),(i, j+1)\}$ é o conjunto dos quatro sítios mais próximos do sítio $(i, j), S_{i, j}^{t}$ e $I_{i, j}^{t}$ são as densidades populacionais dos indivíduos suscetíveis e infectados na geração $t$, antes da movimentação e, $S_{i, j}^{\prime}$ e $I_{i, j}^{\prime}$ representam as densidades populacionais após a movimentação.

As equações que descrevem o processo de interação ou transmissão da doença entre indivíduos suscetíveis e infectados dentro de cada sítio são dadas pelo seguinte sistema de equações a diferenças:

$$
\begin{aligned}
S_{i, j}^{t+1} & =S_{i, j}^{\prime}-\alpha \frac{I_{i, j}^{\prime}}{N} S_{i, j}^{\prime}+\beta\left(N-S_{i, j}^{\prime}\right), \\
I_{i, j}^{t+1} & =I_{i, j}^{\prime}-\beta I_{i, j}^{\prime}+\alpha \frac{I_{i, j}^{\prime}}{N} S_{i, j}^{\prime} .
\end{aligned}
$$

\subsection{Simulações}

Para as simulações do modelo (3.1) a (3.4) é considerado um habitat de $50 \times 50$ sítios, com condições de fronteira reflexivas, ou seja, os indivíduos não atravessam a fronteira, permanecendo no interior do domínio. Iniciamos as simulações considerando toda a população animal suscetível, distribuída uniformemente em todas as células, com apenas dois ratos infectados no centro do reticulado, ou seja, a densidade populacional constitui-se da distribuição uniforme de 100 indivíduos suscetíveis em cada célula do reticulado, exceto na célula $(25,25)$, onde são considerados 2 ratos infectados e 98 ratos suscetíveis. Considera-se também que as taxas de movimentação de indivíduos suscetíveis $\mu_{S}$ e infectados $\mu_{I}$ são iguais, uma vez que os animais infectados não apresentam restrições na movimentação devido à doença.

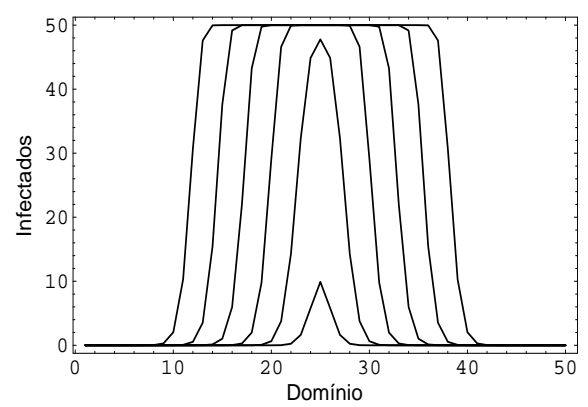

(a)

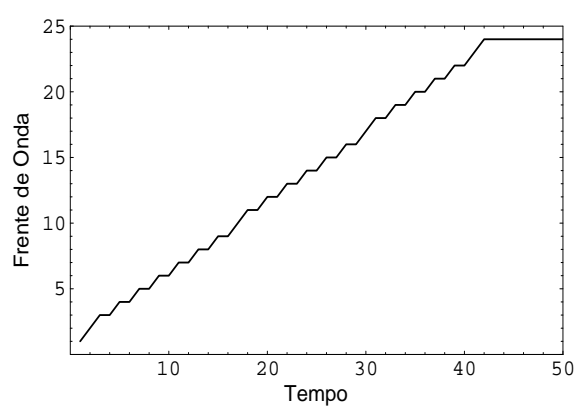

(b)

Figura 3: (a) Perfil da frente de onda de infectados com $\alpha=2, \beta=1$ e $\mu_{S}=\mu_{I}=$ 0,4 nas gerações $t=5,10,15,20,25$ e 30 ; (b) frente de onda.

Em uma primeira simulação consideramos $\alpha=2$ e $\beta=1$, de forma a satisfazer as condições de estabilidade do ponto de equilíbrio endêmico do modelo discreto sem estrutura espacial. Constatamos que nas gerações sucessivas, os indivíduos 
infectados movimentam-se e, através do contato, transmitem a doença para os animais suscetíveis, o que gera uma onda de dispersão da enfermidade. Na Figura 3(a) observa-se a frente de onda dos indivíduos infectados em diferentes instantes de tempo. Após 40 gerações, aproximadamente, as populações de suscetíveis e infectados apresentam uma distribuição espacial homogênea em toda a região considerada.

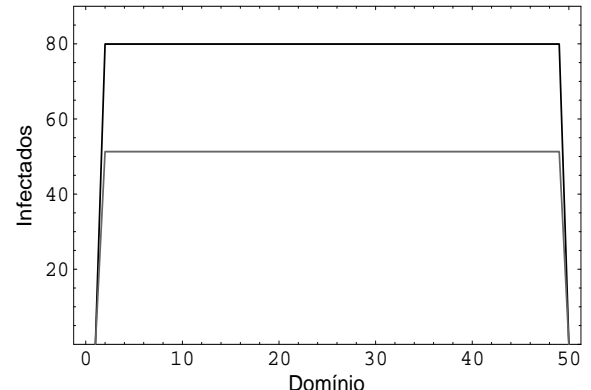

(a)

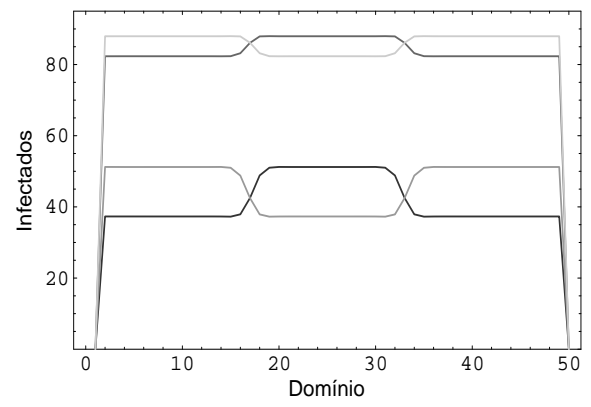

(c)

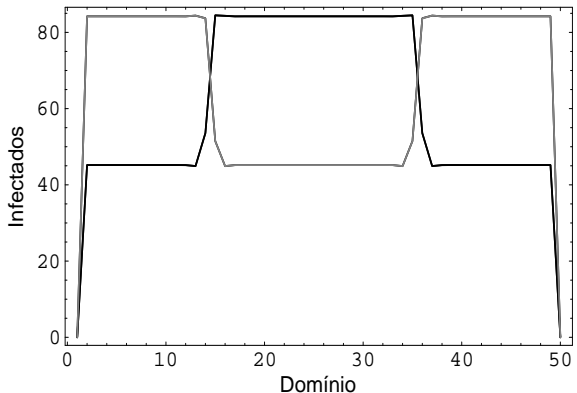

(b)

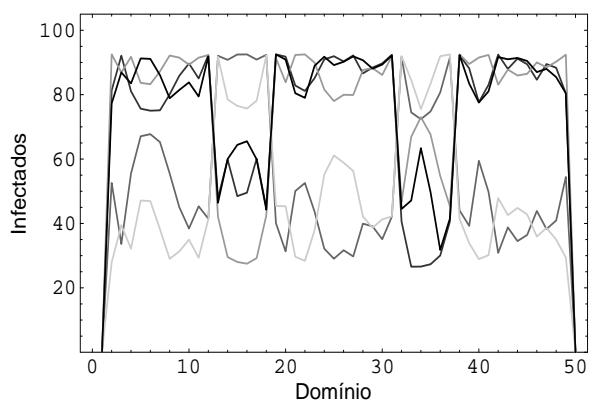

(d)

Figura 4: Distribuição espacial de infectados em um corte do domínio para diferentes tempos, correspondente a: (a) ciclo de período $2, \alpha=3,2$ e $\beta=1$; (b) ciclo de período $2, \alpha=3,4$ e $\beta=1$; (c) ciclo de período $4, \alpha=3,52$ e $\beta=1$ e (d) caos, $\alpha=3,9$ e $\beta=1$.

Se $\widetilde{I}$ denota a densidade limiar abaixo da qual os indivíduos infectados não podem ser detectados, a região na qual $I_{i, j}^{t}$ excede $\widetilde{I}$, isto é, $\Omega_{t}=\left\{i, j: I_{i, j}^{t}>\widetilde{I}\right\}$ será considerada como a região infectada, no tempo $t$. Consideramos $\widetilde{x}^{t}=(\widetilde{i}, \widetilde{j})$ a posição do domínio, na geração $t$, na qual $I_{i, j}^{t}$ intercepta o valor limiar $\widetilde{I} ; \widetilde{x}^{t}$ corresponde à frente da onda de infecção [7]. Como o meio é homogêneo e a difusão isotrópica, fixamos uma direção e identificamos, em cada geração, a posição $\widetilde{x}^{t}$ da frente de onda, obtendo assim uma curva cuja inclinação fornece uma estimativa da velocidade de dispersão da população de infectados. A Figura 3(b) ilustra a variação da frente de onda com o tempo, para $\alpha=2$ e $\beta=1$. Neste caso, a velocidade estimada é de aproximadamente 0,5 sítios/geração. Dados experimentais sobre o comportamento de movimentação dos roedores permitiriam estabelecer as dimensões da malha e assim, calcular a velocidade de espalhamento de uma epidemia. 


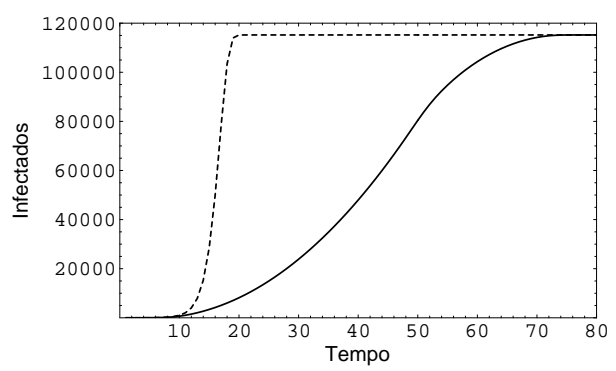

Figura 5: Número total de indivíduos infectados para o modelo sem estrutura espacial (curva tracejada) e para o modelo espacialmente distribuído (curva contínua), $\alpha=2, \beta=1$.

Assim como no modelo sem dispersão, considerando os parâmetros $\alpha$ (taxa de contato) e $\beta$ (taxa de nascimento e mortalidade) fora do domínio da região de estabilidade para o ponto de equilíbrio endêmico, aparecem soluções nas quais as populações totais de infectados e suscetíveis oscilam: surgem ciclos com período 2, período 4 e, assim sucessivamente, até as soluções aperiódicas ou caóticas aparecerem. Às soluções periódicas no tempo, correspondem distribuições espaciais cujo valor em cada ponto, oscila de geração para geração, de acordo com o respectivo período. Quando as populações totais variam caoticamente, a distribuição espacial de infectados e suscetíveis é heterogênea e varia a cada geração. Na Fig. 4(a) ilustramos os resultados da simulação para $\alpha=3,2$ e $\beta=1$; obtivemos ciclos de período 2 homogêneos, isto é, a distribuição espacial da população é homogênea oscilando entre dois valores. Com $\alpha=3,4$ e $\beta=1$ obtivemos ciclos de período 2 porém agora, a distribuição espacial é heterogênea. As densidades populacionais oscilam entre duas distribuições heterogêneas (Fig. 4(b)). Para $\alpha=3,52$, temos um ciclo de período 4. Neste caso, a distribuição espacial oscila entre quatro distribuições espaciais heterogêneas, conforme podemos observar na Fig. 4(c). Aumentando $\alpha$ para $\alpha=3,9$, as soluções oscilam de maneira aperiódica, no tempo e no espaço (Fig. 4(d)).

Uma comparação com o modelo espacialmente homogêneo permite afirmar que o número total de infectados cresce mais lentamente no modelo com movimentação (Figura 5). Isto ocorre porque a transmissão da doença no modelo espacialmente distribuído se dá através de contatos locais, isto é, os indivíduos infectados transmitem a doença somente para indivíduos espacialmente próximos, enquanto, no modelo sem estrutura espacial, todo indivíduo suscetível tem a mesma probabilidade de ser infectado.

\section{Conclusões}

Neste trabalho analisamos o comportamento de um modelo SI discreto espacialmente estruturado do tipo Redes de Mapas Acoplados para ser aplicado a uma epidemia de leptospirose em uma população de ratos. A principal contribuição 
deste trabalho é enfatizar a importância da variável espacial na análise do modelo SI discreto.

O modelo espacialmente distribuído proposto foi explorado através de simulações numéricas. A propagação da epidemia, a partir de um foco de infecção, ocorre na forma de uma onda de invasão. Simulações numéricas indicam que a velocidade de propagação da doença cresce com o aumento da taxa de contato e do coeficiente de movimentação. A distribuição espacial assintótica de suscetíveis e infectados pode ser homogênea, heterogênea e periódica no tempo assim como caótica no espaço e no tempo. Os dados experimentais obtidos por Tassinari e outros [9] mostram a existência de caos espacial e temporal para a leptospirose na cidade do Rio de Janeiro no período de 1996 a 1999, o que indica que o modelo aqui apresentado pode ser usado no estudo desta doença.

Os resultados obtidos mostram que um modelo sem estrutura espacial pode superestimar a velocidade de propagação de epidemias em que o contato dos infectados com os suscetíveis é local (Fig. 5), o que demonstra a importância da variável espacial nos modelos de Epidemiologia.

No sentido de propor estratégias de contenção do avanço ou previsão do surgimento de focos da doença, é necessário incluir a população de humanos ao modelo. No entanto, como a principal fonte de contaminação humana pela leptospirose é o contato com a urina de ratos infectados, os resultados obtidos neste trabalho apontam as Redes de Mapas Acoplados como uma ferramenta útil na modelagem desta interação.

Em trabalhos futuros pretendemos ajustar os parâmetros para um caso específico bem como construir um modelo incorporando a população de humanos. Pretendemos também analisar outros modelos discretos de epidemiologia com estrutura espacial.

Abstract. A spatially structured SI model is proposed in order to describe the spread of a leptospirousis epidemic in a rat population. The behavior of the model solutions regarding epidemic features, such as the velocity of the disease propagation and the spatial distribution of infectives, was analyzed. A Coupled Map Lattice formulation was considered and stable, periodic as well as chaotic solutions were obtained.

\section{Referências}

[1] L.J.S. Allen, Some Discrete-Time SI, SIR, and SIS Epidemic Models, Mathematical Biosciences, 124 (1994), 83-105.

[2] S.M.H. Centenaro, "Um Modelo Matemático Discreto para a Dispersão da Leptospirose em uma População de Ratos", Dissertação de Mestrado, DEFEMUNIJUÍ, Ijuí, RS, 2007.

[3] C.D. De Paula, "Dinâmica Populacional da Leptospirose em Capivaras (Hydrochaeris hydrochaeris) de Vida Livre", Dissertação de Mestrado, Depto. de Medicina Veterinária Preventiva e Saúde Animal, USP, São Paulo, SP, 2003. 
[4] L. Edelstein-Keshet, "Mathematical Models in Biology", Random House, New York, 1988.

[5] M.P. Hassell, H.N. Comins, R.M. May, Spatial structure and chaos in insect population dynamics, Nature, 353, (1991), 255-258.

[6] E.E. Holmes, Basic Epidemiological Concepts in a Spatial Context. In "Spatial Ecology: The Role of Space in Population Dynamics and Interspecific Interactions" (D. Tilman, P. Kareiva, eds.) pp. 111-136, Princeton University Press, Princeton, 1997.

[7] N. Shigesada, K. Kawasaki, "Biological Invasions: Theory and Practice", Oxford University Press, 1997.

[8] R.V. Solé, J. Bascompte, "Self-Organization in Complex Ecosystems", Princeton University Press, Princeton, 2006.

[9] W.S. Tassinari, D.C.P. Pellegrini, P.C. Sabroza, M.S. Carvalho, Distribuição espacial da leptospirose no município do Rio de Janeiro, Brasil, ao longo dos anos de 1996-1999, Cadernos de Saúde Pública, 20, No. 6 (2004), 1721-1729. 